\title{
Individualized Implant: A surgical advice for rotational stability and additional proximal locking of the Fassier-Duval telescoping nail
}

Frank M Schiedel ( $\square$ frank.schiedel@t-online.de)

Technical advance

Keywords:

Posted Date: June 8th, 2020

DOI: https://doi.org/10.21203/rs.2.24232/v3

License: (c) (1) This work is licensed under a Creative Commons Attribution 4.0 International License.

Read Full License 


\section{Abstract}

Background When stabilizing fractures and osteotomies in growing patients with special conditions of the long bones, the use of the Fassier-Duval nail as intended by the manufacturer (Pega Medical, Quebec, Canada) [1], shows that one problem with passive telescopic nails has not yet been solved. No stability in the rotation can be achieved. Postoperative to immobilize with plaster casts are obsolete in patients with osteogenesis imperfecta, as additional osteopenia due to immobilization must be feared. Especially with fractures and osteotomies in proximal femur long leg spica casts are a torture to patients and caregivers.

Methods By experience in different surgical procedures in usage of the Fassier-Duval nailing system a technical solution for rotational stability of the nail as well as for locking of the nail in the proximal and distal physis could be developed.

Results A transverse slot can be cutted in all available diameters of the female parts to make the FD nail rotationally stable. A proximal locking hole can be drilled individually by the surgeon on the operating table. The technical solution to the problems is presented in two exemplary cases in this article.

Conclusions Rotational stability and additional proximal locking options can be achieved by additionally individualization of the Fassier-Duval telescoping nail during surgery.

\section{Background}

In 2003, a new rod became available for clinical use in Canada, the Fassier-Duval Telescopic IM System (FD rod or FD nail) (Pega Medical, Quebec, Canada) [1].

Since then different telescoping intramedullary rods have been used mainly in the management of osteogenesis imperfecta for about fifty years. The complication rates reported in many reviews of the available techniques have been high [2-5]. The advantage of the FD nail, over the traditional BaileyDubow since 2003 was first reported in 2011 [3].

With the two threaded ends a fixation of the two nail parts in epiphyses can be ensured and only a single surgical entry is necessary [4]. In recent publications a high reoperation rate for proximal rod migration and a complication rate up to $40 \%$ because of rod migration, limited telescoping and joint protrusion was found [3].

Postoperative immobilization with plaster casts are obsolete in patients with osteogenesis imperfecta, as additional osteopenia due to immobilization must be feared. Especially with fractures and osteotomies in proximal femur long leg spica casts are a torture to patients and caregivers. FD nails are used in cases with other bone mineralization problems in skeletal immature patients, in cases with pathologial fractures in growing bones and in cases with congenital pseudoarthrosis of the tibia (CPT) after resection, fusion and correction of the deformity for additionally stabilization. 
Cases with protrusions of at least one centimeter on the proximal femur, the proximal tibia in the knee joint or in the proximal humerus with affection of the acromion have been published [2-5]. Further implant developments to the so far published [6,7], which solve the problem of the rotational instability and missing of locking in both epiphysis are necessary. Even a proprietary development with a hexagonal positive locking design and two locking holes in every epiphysis/metaphysis did not come beyond the stage of development [7].

For some reasons FD nails do only need rotational stability for a few weeks till a bony fusion or osteotomy is healed. The implants stay for long time in growing skeletal immature patients with different and heterogeneous bony conditions. Spahn et al. have found that in surgically naive limbs, FD rods were associated with significantly improved probability of survival compared with static implants [8].

The design of the two rods includes a female component and a male component. It is available in five diameters: $3.2,4.0,4.8,5.6$ and $6.4 \mathrm{~mm}$, up to $420 \mathrm{~mm}$ in length. The individual length depends on the anatomical or intraoperatively measured conditions. The outer nail sheath (female part) has to cutted according to the the original surgical technique [1].

The female nail has to be cut with the diamond disc or similar intraoperatively sterile on the table. Cutting the female with a standard surgical rod or wire cutter is not possible. That would lead to an obstruction of normal telescoping.

Different tools are available for the high speed cutter to clean and deburr the edges. Before implantation the user has to insert the male in the female component and verify that there is a smooth telescoping of the parts relative to each other. For that procedure this is an individualized implant and it is technically necessary to finish the implant in the theatre intraoperative by the given anatomical condition of the bone of the patient. This was a very close step to the question that has to be answered in detail in this report.

Purpose was to find a intraoperative reproducable way of achieving rotational stability without external stabilization and a way for locking to an individualized telescopic rod, FD nail.

\section{Methods}

The surgical procedure as intented by the manufacturer is described as following [1]. Some different hints are given: After correction of the deformity and preparing the bone channel the K-wire, that guides the reamer, hast o be removed from the bone channel. With a second K-wire and a ruler it is possible to do a direct measurement of the hole implant length. Be sure to have a x-ray by $\mathrm{C}$-arm that shows the distal end oft the K-wire in the phyis and not penetrating the joint. Measure the length to the edge of the bone at proximal tibia, femur, humerus or wherever your surgical approach and bone entry is. Now you can notice the maximum length of the whole implant. That means the combination of male and female nail part.

Then - in the common description - the K-wire is removed and the male solid nail is placed in the male driver, making sure that the wings of the male solid nail are fitted into the male driver slot. 
The male drivers have the possibility to lock the male component to facilitate maneuvering the nail upon insertion. After insertion of male nail part in the male driver, simply an eccentric ring has to be turned to the lock position.

Then the male solid nail is pushed and threaded down the bone and screwed into the distal epiphysis. For simplification this step can be missed. The same procedure can be done with a solid combination of male and femal part with usage of the canulated female nail driver. But in this way, no positive locking between the arrows of male nail part and the tube of the female nail does exist. The thread of the male part cannot be screwed in the distal physis, it only can be pushed in the distal physis.

The method changing development has to be performed on the table meanwhile the individual preparation of the female nail part. The female part has to be cutted to desired length. The lengths with overlapping with the male part should be as long as anatomical reasonable. The system is, that the rod should telescope for a long time during growth without disconnecting. If the goal of preparing an individual implant is, to cut it as long as for anatomical reasons possible, than it is easy to cut it to a exaclty measured length. This can be arranged with two K-wires and a ruler as desribed above.

Figure 1 shows, how to cut a U-shaped groove in the distal female nail part. This slot has be as long as the arrows of the male nail part. This is about $4-5 \mathrm{~mm}$. The edges have to be deburred exactly to avoid jammed telescoping. Figure 2 shows the result. If the slot is too long, the edges of the slotted distal female part could be demaged thru insertion or they could bend. An overlapping of slot an arrows for at least $4-5 \mathrm{~mm}$ is enough time for a normal bone healing time of 6-8 weeks. After bony consolidation a antirotational mechanism is no longer needed. Figure 3 shows the nail ready to implant with distal antirotational positive locking fitting arrows in slot. Please test smooth telescoping on table before implantation as shown in the video in supplementary material.

Avoid proximal ride out of the male part in the threaded head of the female part, if you intend to lock the nail in the proximal physis in the next step.

The male nail parts have a distal locking hole for a $1.0 \mathrm{~mm} \mathrm{~K}$-Wire. This is challenging to an unexperienced user. But the locking hole makes sense and should be used to avoid penetration of the distal end into the joint. With a lateral exactly positioned $\mathrm{C}$-arm and the magnifying glass, one can use a 2.0 or $2.7 \mathrm{~mm}$ drill to drill a hole in freehand technique in exactly overlapping with the maximum round nail hole. It is much easier to come from a great hole to a narrow hole with a thin wire then to navigate it in two similar narrow holes. If you have a threaded $1.0 \mathrm{~mm}$ K-wire, use it. Figure 4 shows the result of distal locking in an implanted nail. Verify under fluoroscope that the distal thread is not positioned in the growth plate otherwise normal growth may be affected [1]. If there is not enough space as in a lot of tibial cases in OI or CPT, lock the distal end of the male part proximally above the physis in the metaphyseal bone as shown in Figure 4 at the end of a rod changing procedure due to affection of the physis with lack of telescoping. This leads to reduced telescoping effect (only proximal growing of the tibia leads to telescoping) but is mile better than fixation of the physis by screw or K-wires or penetrating the joint. 
If the surgeon likes to have a second proximal locking option, it is possible to create a full locked rotational stable nail. Simple use your sterile single use stainless steel drill from metal- removal rescue set. Sterile metal drills can be ordered by many distributants and manufacturers worldwide. We have good experience with the $2.7 \mathrm{~mm}$ metal drill bit for stainless steel from DePuy Synthes Inc. with a Synthes-Connector for accu machines.

Use the cut block from the high speed cutter to fix the femal nail tight. Then drill the proximal locking hole exactly between the distal two threads of the screwed head. You want to use it in a safe zone, with a perpendicular K-wire that should lock the nail but not penetrate the proximal physis or the joint (if implanted in the tibia). Figure 5 shows the final result after locking the implant proximal with a $2.0 \mathrm{~mm} \mathrm{~K}$ wire.

If you want to remove the implant for any reasons or pull it back a few millimeter before proximal locking, use a needle holder. The original FD nail instruments have no coupling between implant and female nail drivers and no mechanism of sleeve and soul or any other fixation for pulling out the nail.

\section{Results}

Both techniques were developed by the author between 2014 and 2015. The first surgery with proximal locking of the FD nail was performed as part of a nail change in a 9-year-old female patient with CPT. The FD nail previously had migrated proximally and distally into the bone with no telescoping effect. The bone was healed. Thus, there was no need to stabilize the implant in antirotational manner. Prevention of penetration into ankle joint distally and cut out into knee joint or migration thru the physis were the prerequisites to change the implant. Changing the nail diameter to a thicker implant was anatomically impossible. Due to the too flat and in the middle affected distal epiphysis, a metaphyseal locking was accepted distally by means of transverse K-wire thru the original existing hole at the end of the male nail part. Figures 4 and 5 are showing the result intraoperatively.

The first surgery with creation of a rotation stabilizing groove at the distal end was performed after correction and resection of congenital pseudorthrosis of the tibia in a 8 year old boy after fusion axial stabilization by means of FD-nail was aimed at. Because of the very distal fusion, no primary stability could be achieved. The telescopic nail was performed with epiphyseal K-wire locking distally and proximally (with $2.7 \mathrm{~mm}$ metal drill from Synthes for stainless steel) and individual distal slotting of the female nail part for initially rotationally stability for the first weeks to enable wearing of an orthosis. Figure 6 and 7 are showing the preoperative findings in this patient with CPT (El-Rossasy-Paley type 2, Crawford type 4, Anderson dysplastic type, Boyd type 2) in a.p. x-ray. Figure 8 shows postoperative a.p. $x$ ray with proximal locking by $2.7 \mathrm{~mm}$ hole and $2.0 \mathrm{~mm}$ K-wire and Figure 9 shows distal locking and positive locking fitting to the arrows of the male nail part.

\section{Discussion}


A problem of lack of locking possibility in the proximal nail end of telescopic nails can be solved. Thus, the proximal emigration is solvable, in particular on the tibia. The distal locking is technically possible with the FD nails already due to the models with available bore in the distal thread. A proximal hole must be made individually by the surgeon on the operating table. While it was previously only possible to ensure the stability in the rotation after surgery by weeks of wearing plaster [3], with this method description also an initial rotation-stable implantation of the Fassier-Duval nail is possible.

As a negative criticism may be formulated that this technical solution means additional time. However, the time of nailing is not significantly extended, at most in the range of a few minutes, but the implant can be used after drilling in one go at a time. The interlocking with thin K-wire was previously technically challenging, but is no alternative, if a migration of the nail with joint penetration is to be avoided especially at the knee and on the OSG. This is described as a common problem [2-5]. The distal part of the male part already contains a hole for a $1.2 \mathrm{~mm}$ K-wire for locking in the epiphysis distally.

The implantation of the male and female parts in one piece to a suitable length optimizes the design. The otherwise systemimmanente proximal supernatant of the male part is eliminated and a sintering of the osteotomy site resulting in penetration of the nail parts in the adjacent joints also no longer occurs in this technique.

Legal problems do not need to be feared, even if the implant is changed by user. The entire use of the FD nail is creating an individualized implant during surgery, not off label use. The indendet use is to implant a telescoping nail system. To eliminate known and desribed risks, is a bettering. The further modification in the hand of the surgeon should have no new legal meaning here. The manufacturer does not offer an implant ready for use, to which liability in the case of uncertainty would then apply. The implant FD nail can be brought in all cases only by the careful and anatomical individualization with shortening of both nail parts for use in humans. An extended informed consent by patients or caregivers in such interventions is self-evident. The given limits of the telescoping nail remain even with careful application of the modification described here. Breakage of the distal thin K-wires are seen as well as the migration of the subcutaneously bent locking K-wires proximally and distally. Lack of telescoping was seen even without locking or other modifications.

The length of the wings measures about $4 \mathrm{~mm}$, so that for a time to a successful bone healing and maximum growth of $4 \mathrm{~mm}$ a rotational stability of the two telescoping implant parts is given. In cases of spontaneous distraction (axial instability) fixation with an additional K-Wire across the osteotomy site could be helpful. Due to the deburring inside and outside and the intraoperative functional test on the table before implantation, the smooth telescoping can be tested and proved. The transverse slot can be cutted in all available diameters of the female parts to make the FD nail rotationally stable.

\section{Conclusions}

Rotational stability and additional proximal locking options can be achieved by additionally individualization of the Fassier-Duval telescoping nail during surgery 


\section{Declarations}

\section{Ethics approval and consent to participate}

Not applicable

\section{Consent for publication}

Not applicable

\section{Availability of data and material}

All material is available through the author. Please use the supplementary video material online available.

\section{Competing interests}

The author declare that he has no competing interests.

\section{Funding}

No funding was granted.

\section{Authors' Contributions}

Not applicable.

\section{Acknowledgements}

I want to acknowledge the worthful help of my staff. Melanie Horter, Sarah Wacker and Desiree Veltmann assisted the surgical interventions. Ulrich Elsner made the images and videos and prepared it ready to use.

\section{References}

1. Pega Medical QC. Fassier-Duval Telescopic IM System. Surgical Technique 2009;1:1-19, Pega Medical Inc., http://www.pegamedical.com/medias/iw/fassier-duval_surgical_techniques_en.pdf, last accessed at 02/19/2015

2. Bălănescu R, Ulici A, Rosca D, Topor L, Barbu M. Use of minimally invasive (percutaneous) FassierDuval telescopic rod on an 8 year old patient with Lobstein disease. Chirurgia (Bucur). 2013;108:1205

3. Birke O, Davies N, Latimer M, Little DG, Bellemore M. Experience with the Fassier-Duval telescopic rod: first 24 consecutive cases with a minimum of 1-year follow-up. J Pediatr Orthop. 2011;31:458-64

4. Zeitlin L, Fassier F, Glorieux FH. Modern approach to children with osteogenesis imperfecta. J Pediatr Orthop B. 2003;12:77-87 
5. Nicolaou N(1), Bowe JD, Wilkinson JM, Fernandes JA, Bell MJ. Use of the Sheffield telescopic intramedullary rod system for the management of osteogenesis imperfecta: clinical outcomes at an average follow-up of nineteen years. J Bone Joint Surg Am. 2011;93:1994-2000

6. Cho TJ, Choi IH, Chung CY, Yoo WJ, Lee KS, Lee DY. Interlocking telescopic rod for patients with osteogenesis imperfecta. J Bone Joint Surg Am. 2007;89:1028-35.

7. Lakomy J, Vogt B, Gosheger G, Rödl R, Wähnert D, Schiedel F. Comparative Evaluation of a PositiveLocking Antirotation Mechanism in the Design of Telescopic Nails for Patients in the Growth Phase. ASME. Med. Devices. 2017;11:031008-7.

8. Spahn KM, Mickel T, Carry PM, Brazell CJ, Whalen K, Georgopoulos G, Miller NH. Fassier-Duval Rods are Associated With Superior Probability of Survival Compared With Static Implants in a Cohort of Children With Osteogenesis Imperfecta Deformities. J Pediatr Orthop. 2019;39:e392-e396.

\section{Supplemental Information Note}

\section{Supplementary video materials available}

\section{Figures}

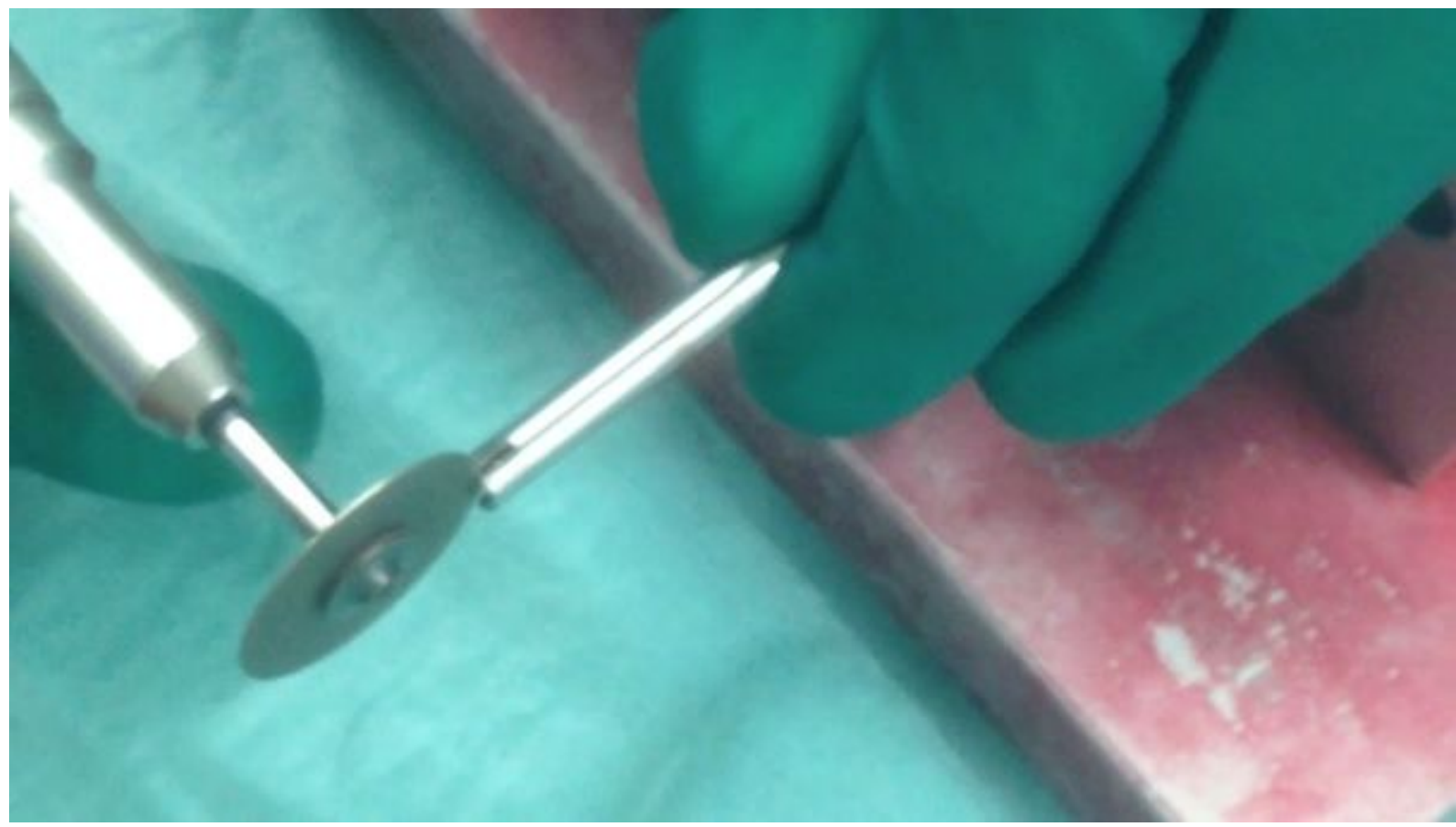

\section{Figure 1}

Slotting of the distal end of female nail part of the FD nail with high speed cutter 


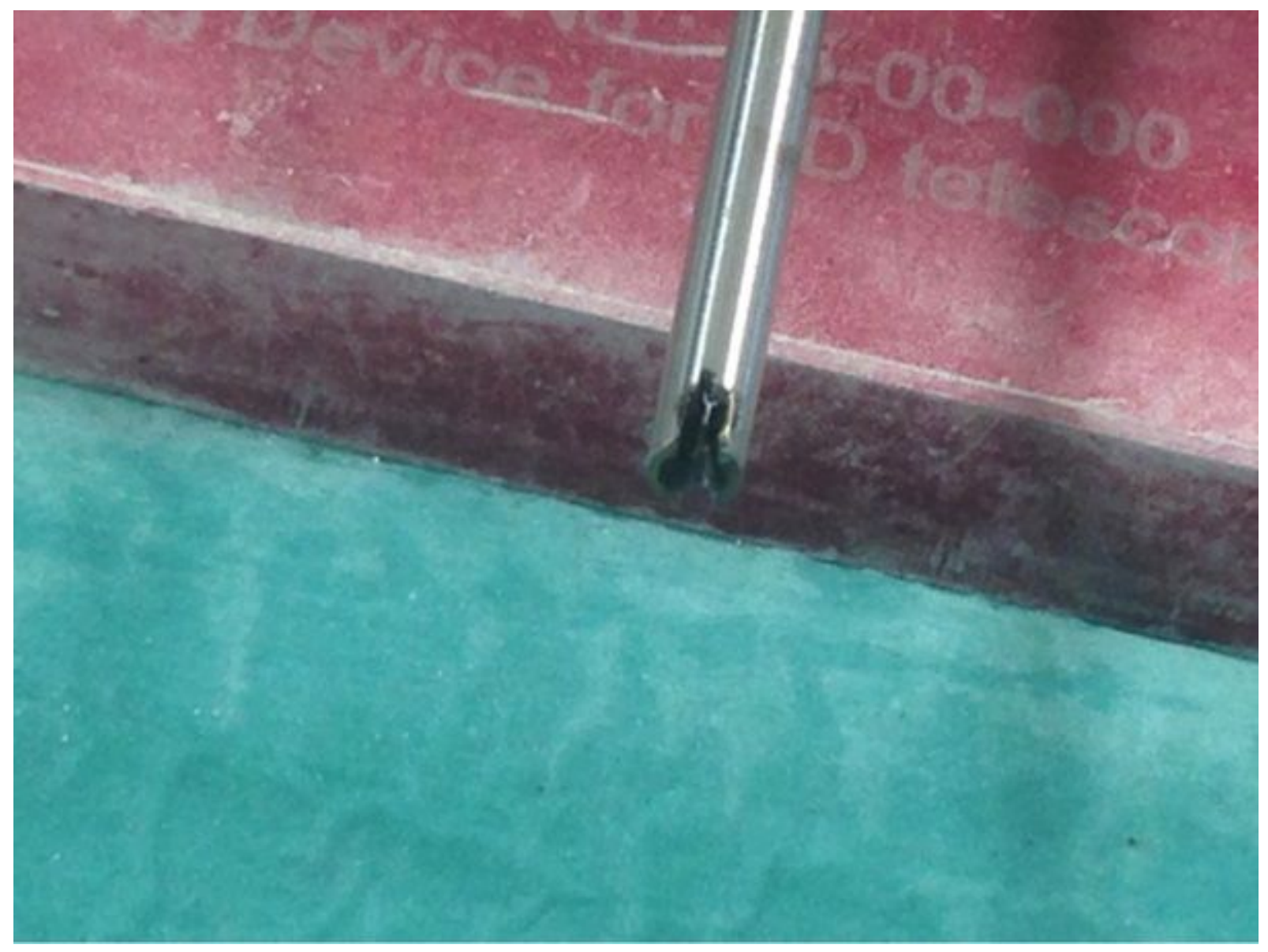

Figure 2

Slot of the distal end of female nail after deburring 


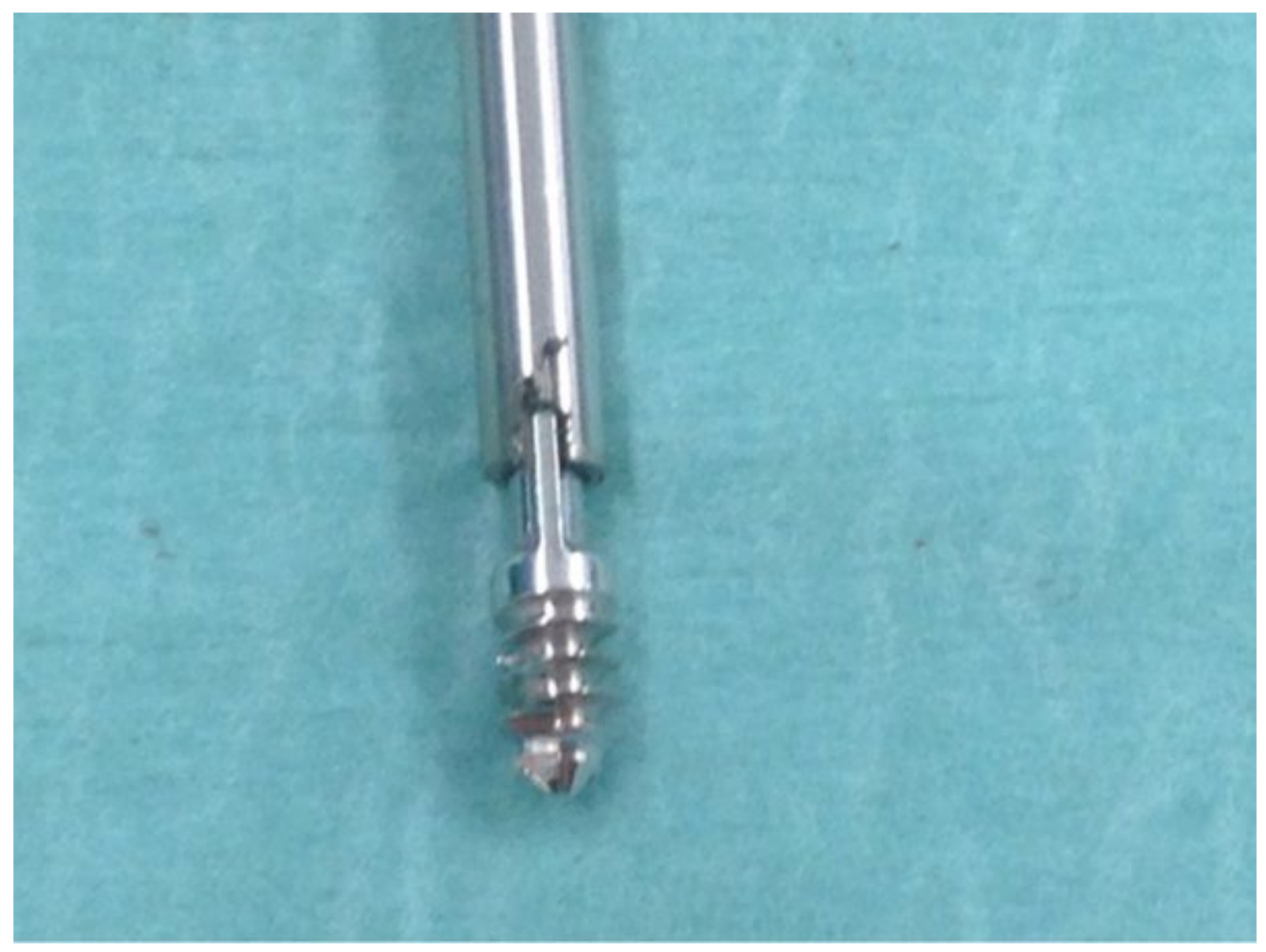

\section{Figure 3}

The slot of the distal end of female nail parts fits together with the arrows of the male nail part for positive locking and rotational stability 


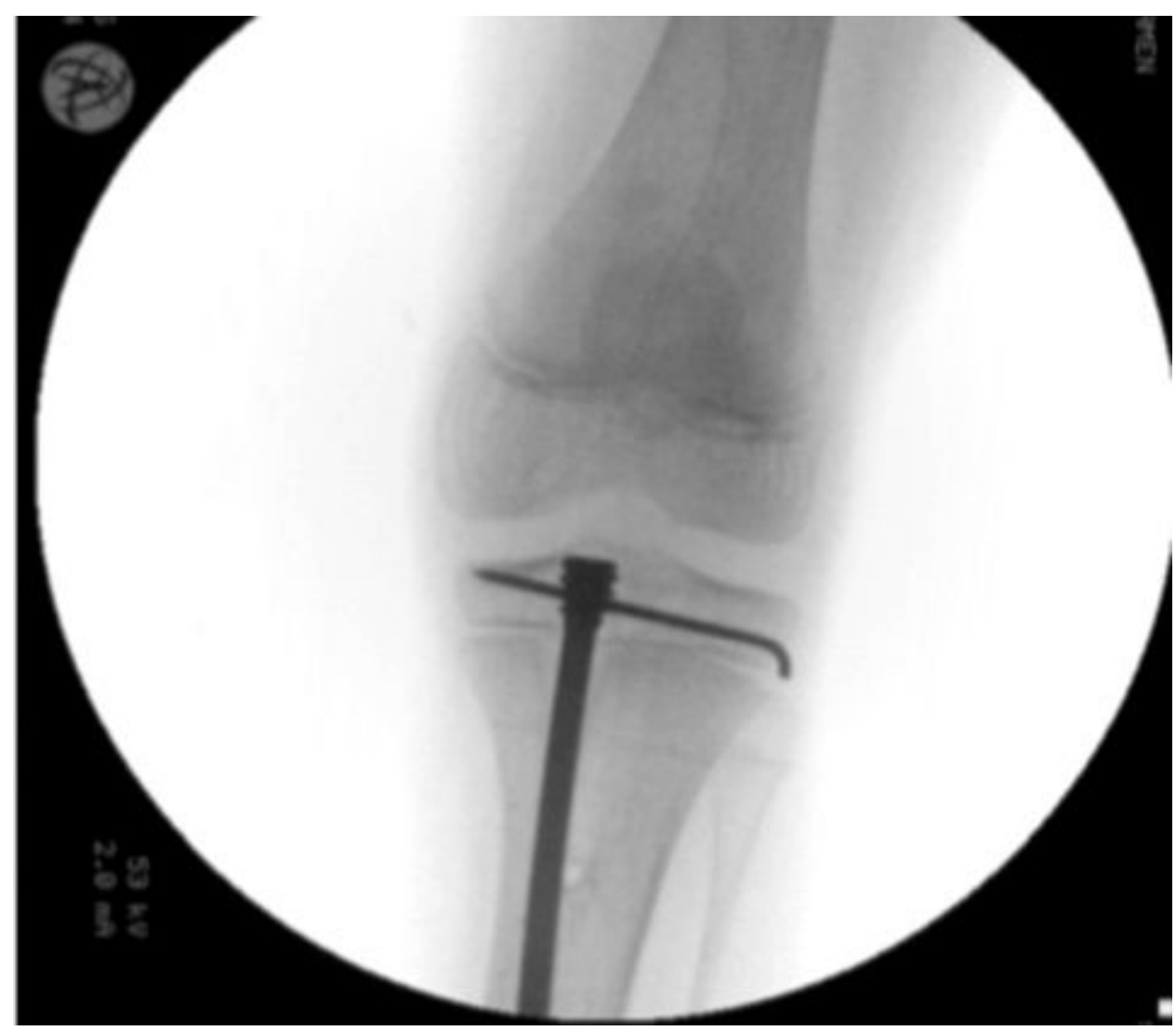

Figure 4

Intraoperative $\mathrm{x}$-ray with $\mathrm{C}$-arm with proximal locking in proximal tibia epiphysis with a $2.0 \mathrm{~mm} \mathrm{~K}$-wire through a self drilled hole in the head of the female nail part with use of a 2.7 stainless steel drill bit. 


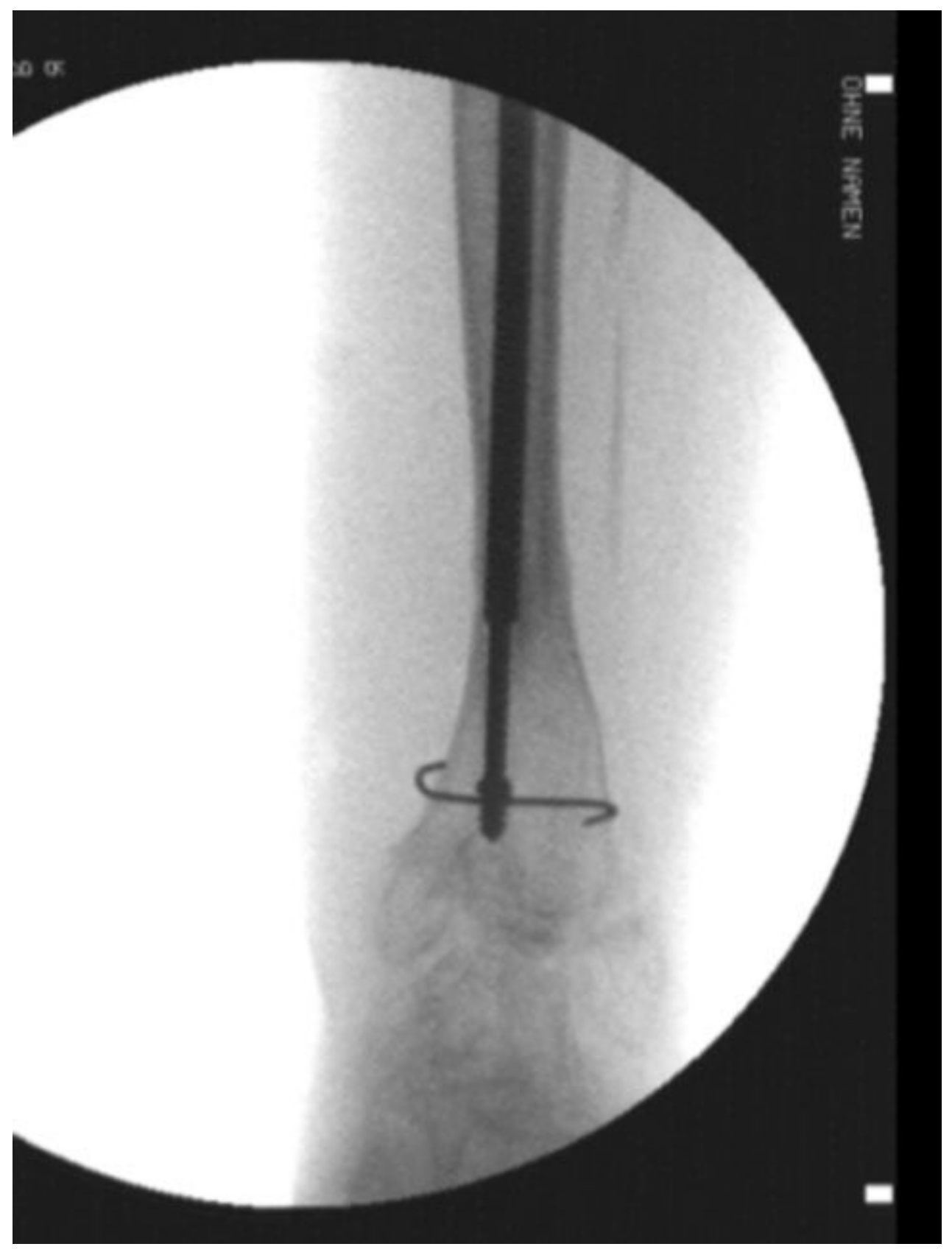

Figure 5

Intraoperative x-ray with C-arm with distal locking thru the threaded end of the FD nail with $1.0 \mathrm{~mm} \mathrm{~K}$-wire. 


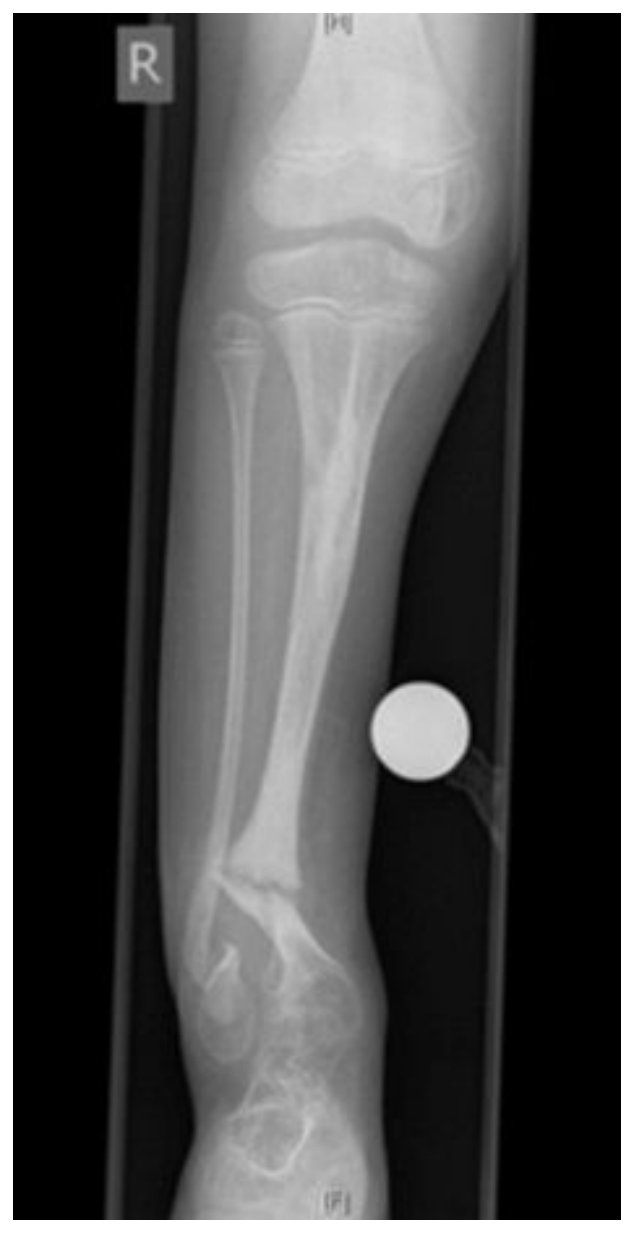

Figure 6

preoperative findings in a 8 years old boy with CPT (El-Rossasy-Paley type 2, Crawford type 4, Anderson dysplastic type, Boyd type 2) in a.p. x-ray 


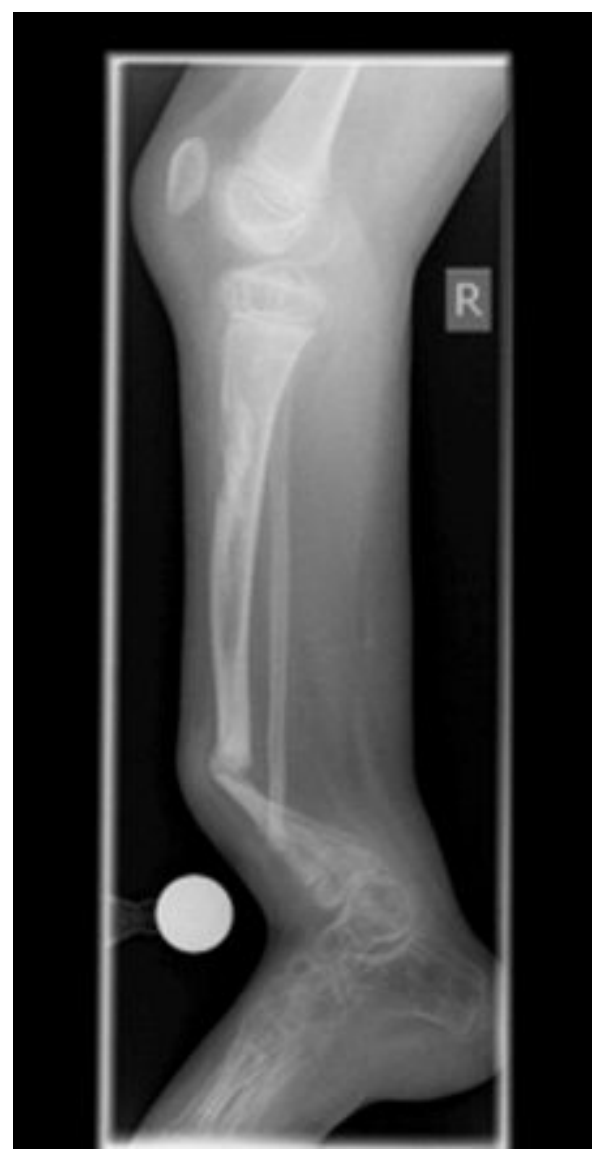

\section{Figure 7}

preoperative findings same patient in lateral $x$-ray

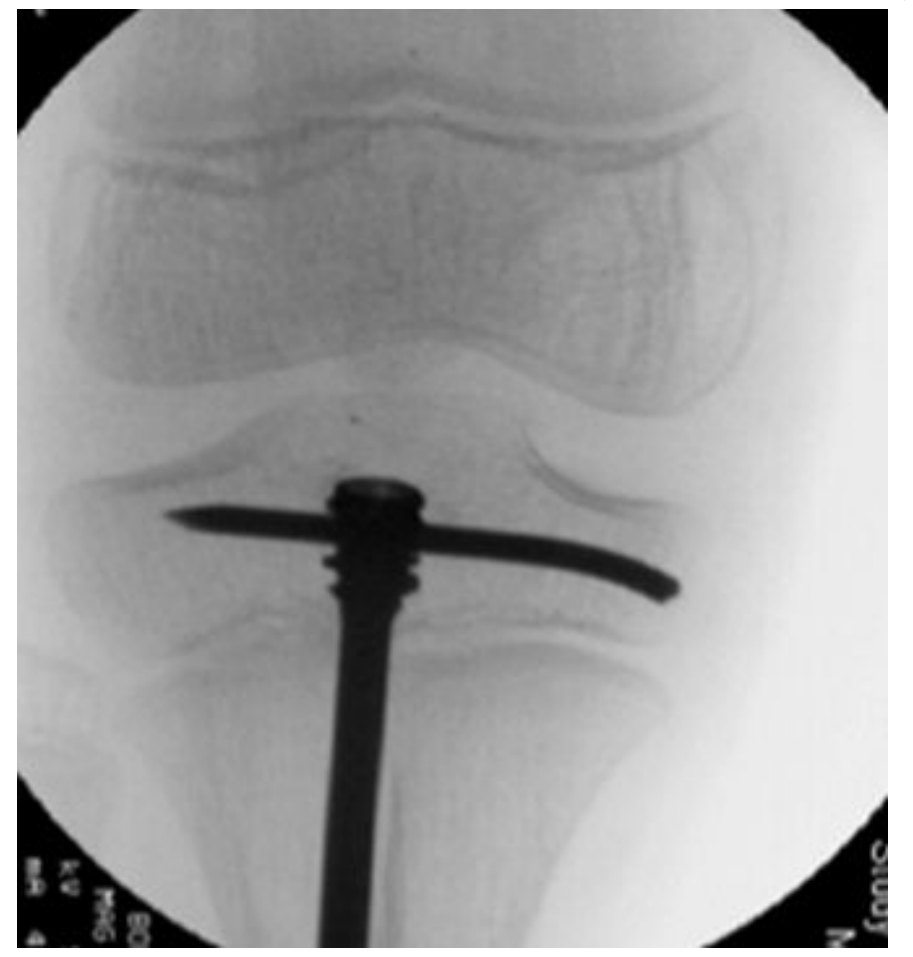

Figure 8 
postoperative a.p. x-ray with proximal locking by $2.7 \mathrm{~mm}$ hole and $2.0 \mathrm{~mm} \mathrm{~K}$-wire

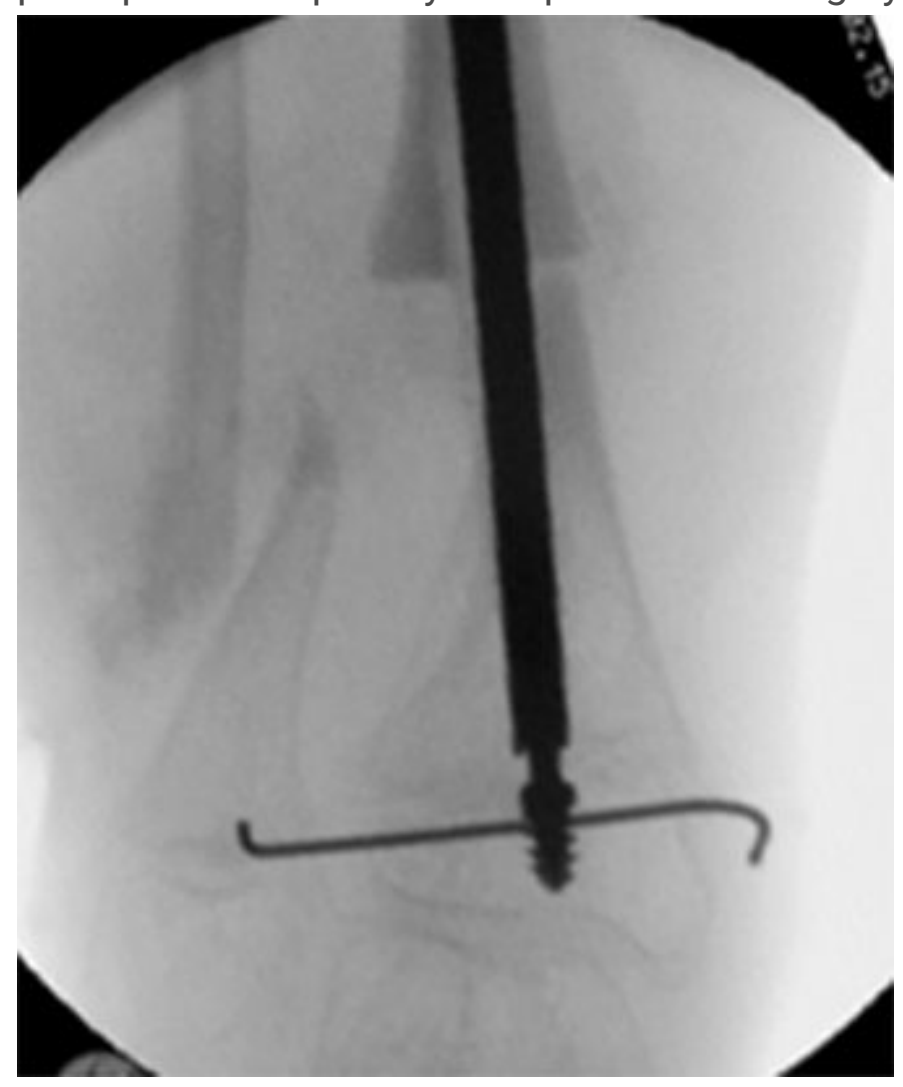

Figure 9

same patient, with distal locking and positive locking fitting to the arrows of the male nail part

\section{Supplementary Files}

This is a list of supplementary files associated with this preprint. Click to download.

- IMG5097.m4v

- vid1.m4v 